Military Technical College Kobry El-Kobbah Cairo, Egypt

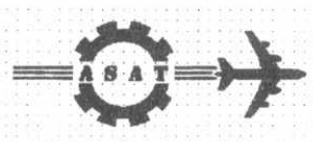

$10^{\text {th }}$ International Conference On Aerospace Sciences\& Aviation Technology

\title{
PHASE SENSITIVE NON-COHERENT MTI RADAR SYSTEM WITH BETTER RADAR RANGE RESOLUTION
}

Sami*. S.S. and Jamil*. M.S.

\section{ABSTRACT}

It is well known [1] that the conventional phase sensitive non-coherent moving target indication [PSN-MTI] system is capable of canceling the moving clutter patch with the following drawbacks; the target plot will be tripled on the radar screen if the target exists within the clutter patch and will be doubled if the target exists at the leading or trailing edge of the clutter patch. So, of course, both the radar range resolution and the radar range measurement accuracy will be affected and become worse by a factor of 3 in the first case and by a factor of 2 in the second case, what is not desired in most modern radars.

In this work, a PSN-MTI system is proposed. It cancels the moving clutter patch, but with doubled target plot width on the screen, not tripled, if target exists within clutter batch. And if the target is at the leading or trailing edge of the clutter patch, the target plot remains as the clutter-free case and not doubled as the conventional system. In such cases, the proposed system will improve both radar range resolution and range measurement accuracy

\section{KEY WORDS:}

Radar,

Moving clutter,

Noncoherent MTI,

phase sensitive noncoherent MTI,

Chaff,

\footnotetext{
* Dr.Prof., Asad academy For Military Engineering, Aleppo, Syria.

*Assistant Prof., Asad academy For Military Engineering, Aleppo, Syria.
} 


\section{NOMENCLATURE}

$T$-pulse repetition interval.

$F=\frac{1}{T}-$ pulse repetition frequency.

$\tau$-pulse duration.

$f_{I F}$-intermediate frequency.

$F_{\text {d.cl. }}$ - clutter Doppler frequency.

$F_{\text {d.tar. }}$-target Doppler frequency.

$\lambda$-transmitted signal wavelength.

$R_{\text {tar. }}$ - initial target distance.

$R_{c l}$ - initial clutter cell distance.

$\varphi_{n}$-transmitter random initial phase in the nth period interval.

$\varphi_{\text {ref.cl. }}$ - clutter reflection phase.

$\varphi_{\text {ref.tar. }}-$ target reflection phase.

\section{I- INTRODUCTION}

In spite of the moving target detection (MTD) system is widely used in most modern radar systems, many different types of MTI system which use the delay-line cancelers, instead of Doppler filter-banks, are still important and necessary in many cases of radar operation conditions. That is, because:

- for the blind speeds to be reduced, the MTD system requires the pulse repletion interval to be changed from returned pulses group to another group, not from pulse to pulse as in the MTI system [2],[3],[4],[5]. In this case, it is possible to loss the group of pulses corresponding to the existed blind speed. So, as a result, the target detection probability will be reduced in those radars used this system as mentioned by [4]. "If the consequences of blind speeds, when MTD is used, are not dealt with, particularly in air traffic control radars, they can become a positive menace to safety". - the MTD system is highly affected by noncoherent pulse interference. When such interference is received simultaneously with one or more target returns, the spectrum of returned pulses becomes wider so as to pass through more than one Doppler filter, what give rise to the target detection probability to be reduced and the target relative velocity to be correctly undetermined.

- the MTD system is very affected by both the radar transmitted and received pulses instability (for example if there is rapid radar cross section changes through the time on the target when the target is maneuvering), what also give rise to the target detection probability to be reduced and the target relative velocity to be correctly undetermined. by the same reason as it was said above for the pulse interference.

- In general, that all radar MTI systems, including the phase sensitive noncoherent moving target indication [PSN-MTI], are less influenced by those probiems than the radar MTD system. So, it is important to improve the radar PSN-MTI performance, including the range resolution and range measurement accuracy to be as high as possible, what is partially done in this work, where the range resolution and range accuracy become higher once and half if the target exists within the clutter patch and higher twice if the target exists at the leading or trailing edge of the clutter patch 
regardless of possible Doppler frequencies of the moving target and clutter and regardless of the number of clutter sources as it will be seen later through the theoretical and the computer simulation sections.

\section{II- THEORETICAL INTRODUCTION}

The block diagram of the conventional PSN-MTI system is shown in Fig.1, and the block diagram of the proposed PSN-MTI system is shown in Fig.2. It is obvious, from these two figures that the two systems are very similar in their operation principle. The main difference, may be said, between the two systems is included in, which range resolution cells must be applied to the main and reference phase detector so as to cancel the hole clutter patch with the target signal to be doubled (not tripled) within the clutter and singled (not doubled) at the leading or trailing edge of the clutter.

We will demonstrate how this is may be done through the proposed system in the case if the target exists within the moving clutter patch and the non-coherent radar (with random periodic transmitted signal phase) is used.

Let the target signal exists in the $\mathrm{k}^{\text {th }}$ resolution cell at the $\mathrm{n}$ period repetition interval of the $\mathrm{N}$ returned pulses from the target. And let be assumed that both the target and the clutter are approaching, and also assumed that target and all clutter cells distance phases are $\frac{4 \pi R_{\text {tar. }}}{\lambda}=\frac{4 \pi R_{c l}}{\lambda}=$ constant and equal to zero for the simplicity of writing the system performance equations.

It is seen from Fig.2, that:

First:

the clutter $(k-2)^{\text {th }}$ cell signal at the main phase detector channel is:

$x_{k-2, n}(t)=X_{k-2, n}(t) \exp j\left(2 \pi f_{I F} t+2 \pi F_{d . c l .} n T+\varphi_{n}+\varphi_{k-2, n, r e f . c l .}\right)$

(assuming here, that the target is approaching and the distance phase $\frac{4 \pi R_{c l} \text {. is zero) }}{\lambda}$ and the sum signal of clutter $(\mathrm{k}-1)^{\text {th }}$ cell signal and clutter $(\mathrm{k}-2)^{\text {th }}$ cell signal at the reference phase detector channel is:

$$
\begin{aligned}
\dot{x}_{\text {sum }, k-1, k-2, n}(t)= & \dot{x}_{k-1, n}(t)+\dot{x}_{k-2, n}(t) \\
= & X_{k-1, n}(t) \exp j\left[2 \pi f_{I F} t+2 \pi F_{d . c l .}(n T-\tau)+\varphi_{n}+\varphi_{k-1, n, r e f . c l .}\right]+ \\
& +X_{k-2, n}(t) \exp j\left(2 \pi f_{I F} t+2 \pi F_{d . c l .} n T+\varphi_{n}+\varphi_{k-2, n, r e f . c l .}\right) \\
& =X_{\text {sum, } k-1, k-2, n}(t) \exp j\left(2 \pi f_{I F} t+2 \pi F_{d . c l .} n T-2 \pi F_{d . c l .} \tau+\varphi_{n}+\right. \\
& \left.+\varphi_{k-1, n, r e f . c l .}+\varphi_{k-2, n, r e f . c l .}\right)
\end{aligned}
$$

then, the clutter $(k-2)^{\text {th }}$ cell phase detector output is: 
$x_{k-2, n, o u t}(t)=X_{k-2, n}(t) \exp j\left(-2 \pi F_{d . c l .} \tau+\varphi_{k-1, n, \text { ref.cl. }}\right)$

By the same way we can find that:

$$
x_{k-2, n+1, \text { out }}(t)=X_{k-2, n+1}(t) \exp j\left(-2 \pi F_{d . c l .} \tau+\varphi_{k-1, n+1, r e f . c l}\right)
$$

at the phase detector output in the $(n+1)^{\text {th }}$ period repetition interval and so on for the $(n+2)^{\text {th }} ;(n+3)^{\text {th }}$; ; $(\mathrm{N}-1)^{\text {th }}$ period.

It is seen from (3) and (4) that the periodic signals

$x_{k-2,1, \text { out }}(t) ; x_{k-2,1, \text { out }}(t) ; \ldots \ldots \ldots . . ; x_{k-2, N-1, \text { out }}(t)$ are modulated by zero Doppler frequency, considering that the $\varphi_{k-1, n, \text { ref.cl. }} \approx \varphi_{k-1, n+1, \text { ref.cl. }}=$ cons tant

and the $X_{k-2, n}(t) \approx X_{k-2, n+1}(t)=$ constant

So, it is possible then to cancel the clutter $(\mathrm{k}-2)^{\text {th }}$ by using the well known single or more order delay-line canceler.

\section{Second:}

the clutter $(\mathrm{k}-1)^{\text {th }}$ cell signal at the main phase detector channel is:

$x_{k-1, n}(t)=X_{k-1, n}(t) \exp j\left(2 \pi f_{I F} t+2 \pi F_{d . c l .} n T+\varphi_{n}+\varphi_{k-1, n, \text { ref.cl. }}\right)$

and the sum signal of target $(k)^{\text {th }}$ cell signal and clutter $(k-1)^{\text {th }}$ cell signal at the reference phase detector channel is:

$$
\begin{aligned}
x_{s u m, k, k-1, n}(t)= & x_{k, n}(t)+x_{k-1, n}(t) \\
& =x_{k, n, t a r .}(t)+x_{k, n, c l .}(t)+x_{k-1, n}(t) \\
& =X_{k, n}(t) \exp j\left[2 \pi f_{I F} t+2 \pi F_{d . t a r .}(n T-\tau)+\varphi_{n}+\varphi_{k, n, \text { ref.tar. }}\right]+ \\
& +X_{k, n}(t) \exp j\left[2 \pi f_{I F} t+2 \pi F_{d . c l .}(n T-\tau)+\varphi_{n}+\varphi_{k, n, r e f . c l .}\right]+ \\
& +X_{k-1, n}(t) \exp j\left(2 \pi f_{I F} t+2 \pi F_{d . c l .} n T+\varphi_{n}+\varphi_{k-1, n, r e f . c l .}\right) \\
& =X_{s u m, k, k-1, n}(t) \exp j\left(2 \pi f_{I F} t+2 \pi F_{d . c l .} n T-2 \pi F_{d . c l .} \tau+\right. \\
& +2 \pi F_{d . t a r .} n T-2 \pi F_{d . t a r .} \tau+\varphi_{n}+\varphi_{k, n, r e f . t a r .}+ \\
& \left.+\varphi_{k, n, r e f . c l .}+\varphi_{k-1, n, r e f . c l .}\right)
\end{aligned}
$$

then, the clutter $(k-1)^{\text {th }}$ cell phase detector output is:

$$
\begin{aligned}
x_{k-1, n, \text { out }}(t) & =X_{k-1, n}(t) \exp j\left(2 \pi F_{d . t a r .} n T-2 \pi F_{d . t a r .} \tau+\right. \\
& \left.+\varphi_{k, n, \text { ref.tar }}-2 \pi F_{d . c l .} \tau+\varphi_{k, n, \text { ref.cl. }}\right)
\end{aligned}
$$


By the same way we can find that:

$$
\begin{aligned}
x_{k-1, n+1, \text { out }}(t) & =X_{k-1, n+1}(t) \exp j\left(2 \pi F_{d . t a r .}(n+1) T-2 \pi F_{d . t a r .} \tau+\right. \\
& \left.+\varphi_{k, n+1, \text { ref.tar }}-2 \pi F_{d . c l .} \tau+\varphi_{k, n+1, \text { ref.cl. }}\right)
\end{aligned}
$$

at the phase detector output in the $(n+1)^{\text {th }}$ period repletion interval and so on for the $(n+2)^{\text {th }},(n+3)^{\text {th }}$; ; $(\mathrm{N}-1)^{\text {th }}$ periods.

It is seen from (7) and ( 8 ) that the periodic signals:

$x_{k-1,1, \text { out }}(t) ; x_{k-1,2, \text { out }}(t)$;. $; x_{k-1, N-1, \text { out }}(t)$ are modulated by the target Doppler frequency $F_{\text {d.tar. }}$, considering that:

$\varphi_{k, n, \text { ref.cl. }} \approx \varphi_{k, n+1, \text { ref.cl. }}=$ cons tant $; \varphi_{k, n, \text { ref.tar. }} \approx \varphi_{k, n+1, \text { ref.tar. }}=$ cons tant

and $X_{k-1, n}(t) \approx X_{k-1, n+1}(t)=$ constant

So, the $(k-1)^{\text {th }}$ cell would not be canceled by the delay-line canceler.

Exactly, by the same way we can find that the signals:

$\dot{x}_{k, 1, \text { out }}(t) ; \dot{x}_{k, 2, \text { out }}(t) ; \ldots \ldots \ldots . . ; x_{k, N-1, \text { out }}(t)$ are also modulated by the target Doppler frequency $F_{d . t a r}$, and therefore also would not be canceled.

But, the $(\mathrm{k}+1)^{\text {th }}$ cell signals will be canceled as well as the $(\mathrm{k}-2)^{\text {th }}$ cell signals by the same reason, which is:

$\dot{x}_{k+1,1, \text { out }}(t) ; \dot{x}_{k+1,2, \text { out }}(t) ; \ldots \ldots \ldots . . ; \dot{x}_{k+1, N-1, \text { out }}(t)$ are modulated by zero Doppler frequency.

It is necessary In this system, it is necessary to add a threshold level circuit at $3 \sigma_{\text {rec.noise }}$ following the amplitude detector so that the target signal would not be canceled when the target exists outside the clutter patch. As a result, we can see that the target signal is present only at both the $(k-1)^{\text {th }}$ and $k^{\text {th }}$ cells, not at $(k-1)^{\text {th }}$ and $\mathrm{k}^{\text {th }}$ and $(\mathrm{k}+1)^{\text {th }}$ as in the conventional system.

It is possible, also by the same way theoretically indicate that the target signal is single (not doubled as in the conventional system) if the target is existed at the leading or trailing edge of the clutter patch.

In addition to these results we can also note that the PSN-MTI system is not affected by the transmitter phase instability as shown by (3),(4),(7) and (8).

\section{III- COMPUTER SIMULATION}

The MathCAD program is used to simulate a noncoherent radar with periodically random initial transmitted phase $\varphi_{n}$ and with the following parameters and radar operation conditions:

- pulse repetition interval of 600 time unit $(T=600)$.

- pulse duration of 40 time unit $\left(\tau=40\right.$ ). So, there are $\frac{T}{\tau} K=15$ range

resolution cells.

- moving clutter is extended from $\mathrm{k}=2 \tau=80$ to $\mathrm{k}=10 \tau=400$. 
- first moving target exists at the clutter leading edge; in $\mathrm{k}=\tau=40$.

- second moving target exists within the clutter; in $\mathrm{k}=6 \tau=240$.

- third moving target exists at the clutter trailing edge; in $\mathrm{k}=10 \tau=400$.

- fourth moving target exists outside the clutter patch; in $\mathrm{k}=13 \tau=520$.

- the receiver noise is normal distributed with mean $(\eta=0)$, and standard deviations $(\sigma=0.05 ; 0.1)$ of target signal amplitude.

- the number of return pulses in the 3-dB antenna beam width is $N=3$.

- the clutter and target phase reflection is constant from pulse to pulse.

Considering these conditions, both the conventional and proposed systems will be simulated in the following four cases:

Case (a):

- target signal amplitude level to receiver noise amplitude level ratio is: $\frac{X_{\text {tar. }}}{X_{\text {n.rec. }}}=20$.

- clutter signal amplitude level to target signal amplitude level ratio is: $\frac{X_{c l}}{X_{t a r}}=1$.

- one source moving clutter with Doppler frequency $F_{\text {d.cl. }}=\frac{F}{10}$.

- targets Doppler frequencies are: $F_{\text {d.tar. } 1}=F_{\text {d.tar } .2}=F_{\text {d.tar. } 3}=F_{\text {d.tar } .4}=\frac{F}{2}$.

Output signals of the single delay-line in the simulated conventional and proposed systems are shown is in Fig.3, Fig. 4 respectively.

Case (b):

Is the same as the case (a) except that the clutter signal amplitude level to target signal amplitude level ratio is: $\frac{X_{c l}}{X_{\text {tar. }}}=3$.

Output signals of the single delay-line in the simulated conventional and proposed systems are shown is in Fig.5, Fig.6 respectively.

\section{Case (c):}

Is the same case (a) except that a moving clutter source with Doppler frequency $F_{\text {d.cl. }}=\frac{F}{6}$ is added

- the considered targets Doppler frequencies are:

$$
F_{\text {d.tar } .1}=\frac{F}{4} ; F_{\text {d.tar } .2}=\frac{F}{3} ; F_{\text {d.tar } .3}=\frac{F}{3} ; F_{\text {d.tar } .4}=\frac{F}{4} \text {. }
$$

Output signals of the single delay-line in the simulated conventional and proposed systems are shown is in Fig.7, Fig.8 respectively.

\section{Case (d):}

is the same as case (a) except that, there are three clutter sources: 
- first clutter source is extended from $\mathrm{k}=5 \tau$ to $\mathrm{k}=80 \tau$ with $F_{d . c l}=0$.

- second clutter source is extended from $\mathrm{k}=10 \tau$ to $\mathrm{k}=50 \tau$ with $F_{\text {d.cl. }}=\frac{F}{15}$.

- third clutter source is extended from $\mathrm{k}=5 \tau$ to $\mathrm{k}=80 \tau$ with $F_{\text {d.cl. }}=\frac{F}{10}$.

Output signals of the single delay-line in the simulated conventional and proposed systems are shown is in Fig.9, Fig.10 respectively.

The simulated results show that in the proposed system, the target plot is doubled (not tripled as in the conventional system) if the target exists within the clutter patch and the targei plot is single (not doubled as in the conventional system) if the target is at the leading or tailing edge of the clutter patch. This means that the radar range resolution and range measurement will be better by using the proposed PSN-MTI system instead of the conventional system.

\section{IV- CONCLUSIONS}

- the proposed phase sensitive noncoherent moving target indication system is able to cancel the multi-source fixed and/or moving clutter patch as the conventional system with the target plot on the screen is doubled (not tripled as in the conventional system) when the target exists within the clutter patch. And if the target is at the leading or tailing edge of the clutter patch. Its plot is not doubled as in the conventional system. So, as a consequence, both the radar range resolution and range measurement will be improved because the target plot on the screen is decreased by a factor of 1.5 when the target exists within the clutter patch and by a factor of 2 when the target is at the leading or tailing edge of the clutter patch.

- all the results are obtained through the simulation, using MathCAD program, of the conventional and proposed systems under the most real radar operating conditions.

\section{REFERENCES}

[1] Skulking, M.I., "Radar Handbook", McGraw-Hill Book Company, N. Y.,(1970).

[2] Skolnik, M.I., "Radar Handbook", 2d ed., McGraw-Hill Publishing Company, N.Y., (1990).

[3] Skolnik, M.I., "Introduction to Radar Systems", 2d ed., McGraw-Hill Publishing International Company, N.Y., (1990).

[4] Cole, H.W.,"Understanding Radar",2d ed., Blackwell Scientific Publications, Oxford, (1992).

[5] Shearman, E. D. R., Bradsell, P., "Advanced Radar Techniques And Systems", British Library Cataloguing in Publication Data, (1993). 


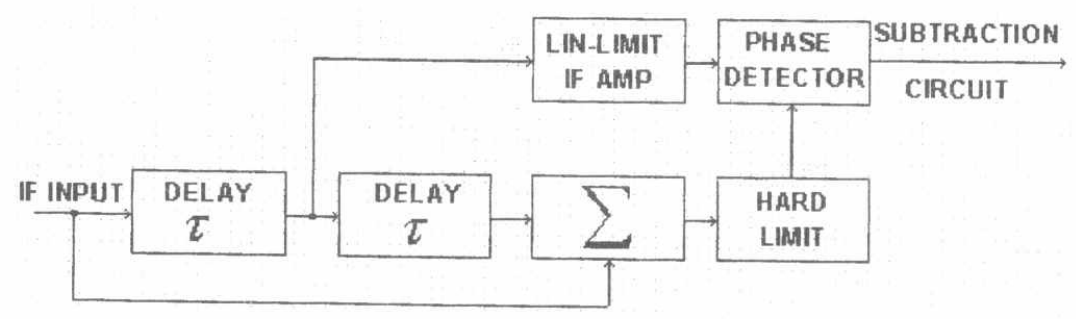

Fig.1. The conventional phase sensitive noncoherent MTI system.

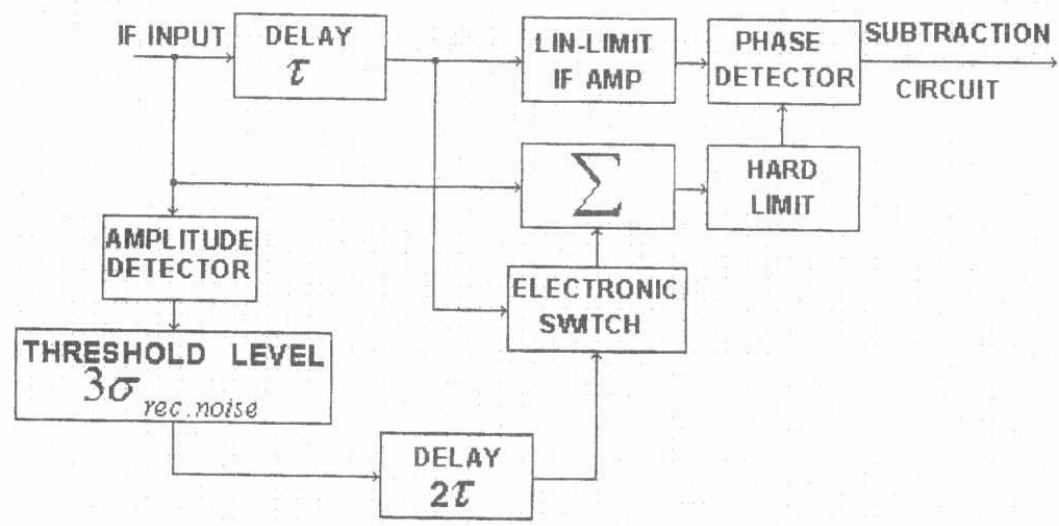

Fig.2. The proposed phase sensitive noncoherent MTI system.

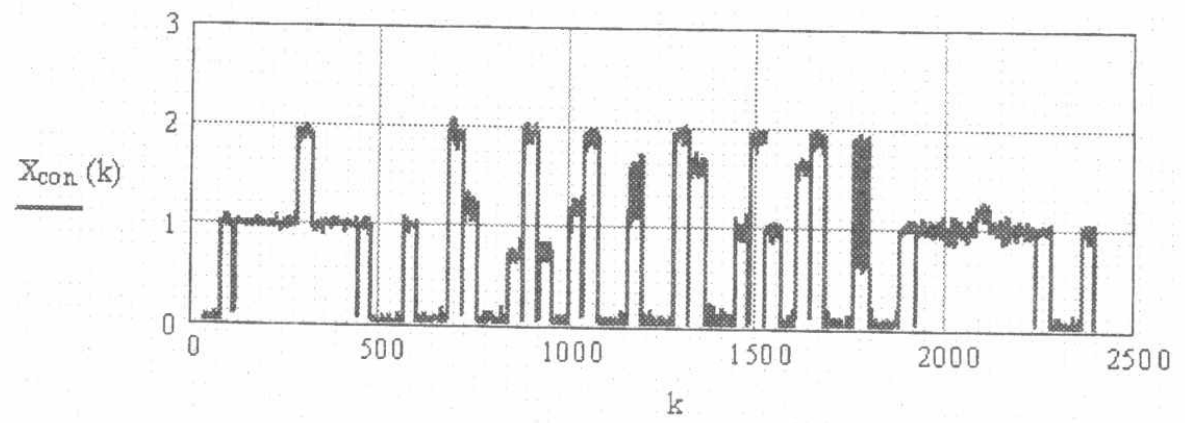

Fig. 3. Single delay-line canceler output signal of the simulated conventional system. [ case (a)] 


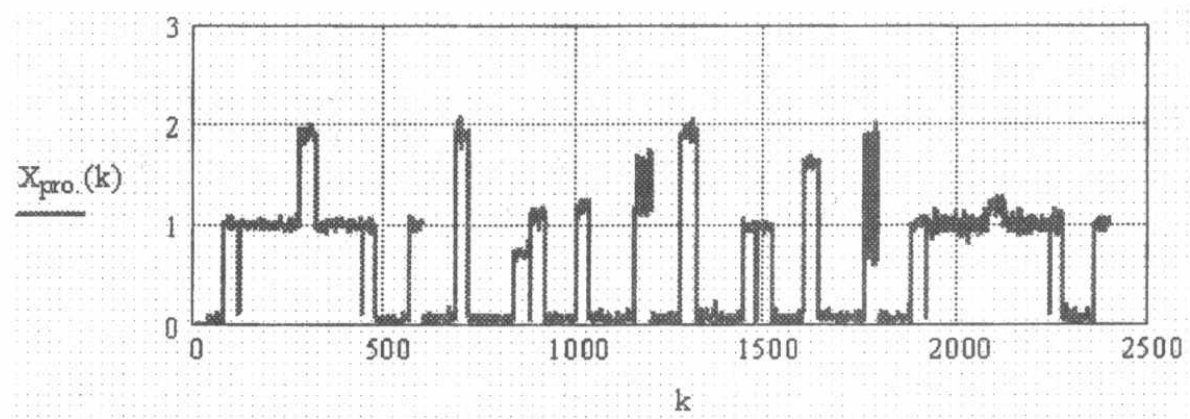

Fig.4. Single delay-line canceler output signal of the simulated proposed system. [ case (a)]

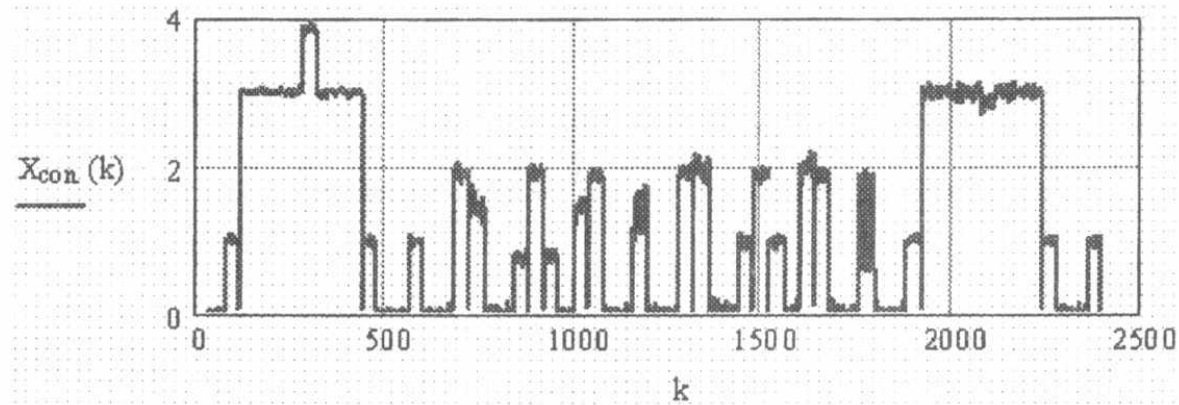

Fig.5. Single delay-line canceler output signal of the simulated conventional system. [ case (b)]

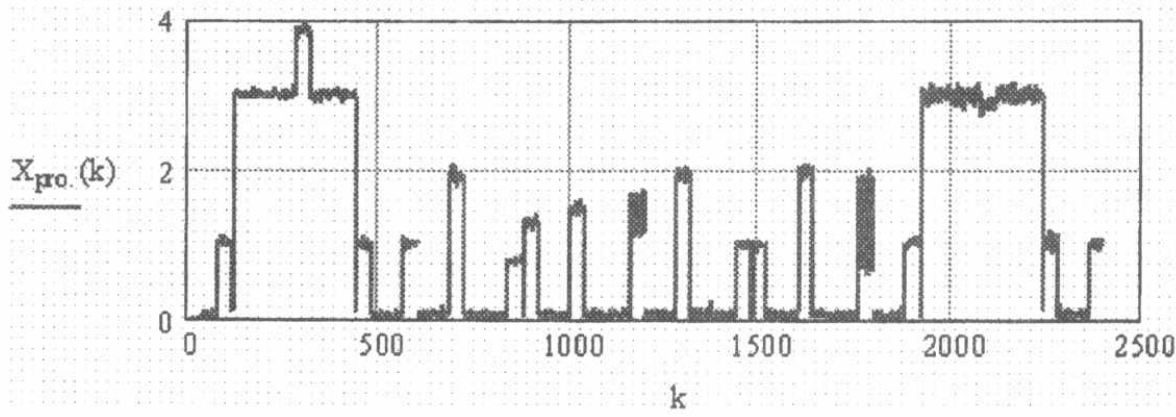

Fig.6. Single delay-line canceler output signal of the simulated proposed system. [ case (b) ] 


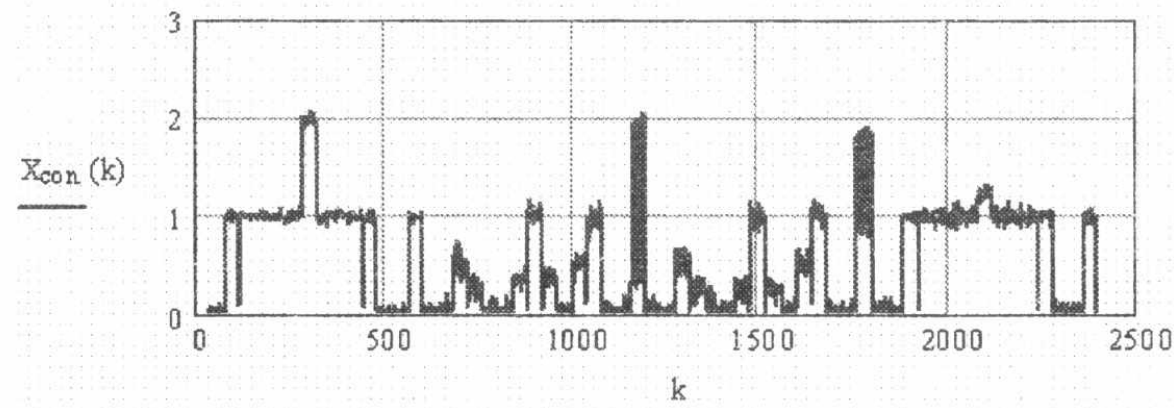

Fig.7. Single delay-line canceler output signal of the simulated conventional system. [ case (c) ]

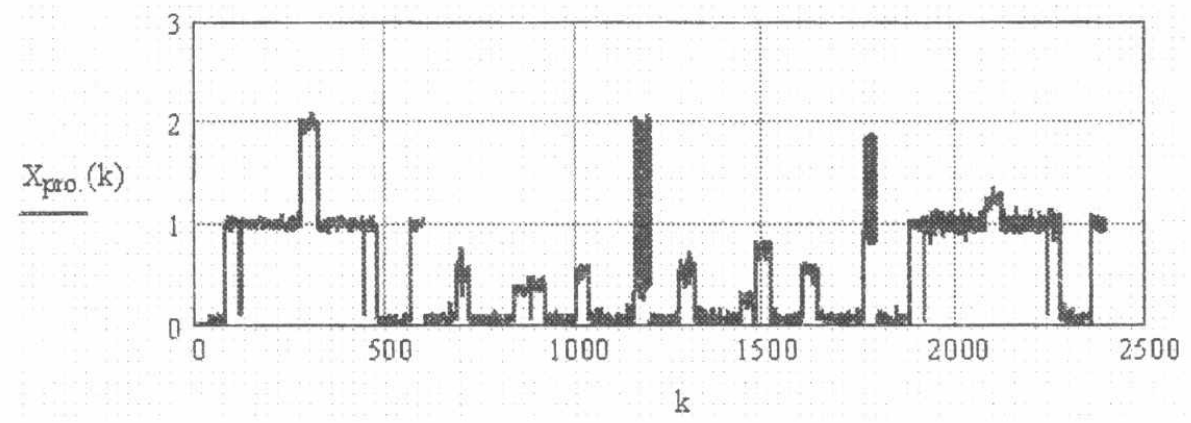

Fig.8. Single delay-line canceler output signal of the simulated proposed system. [ case (c) ]

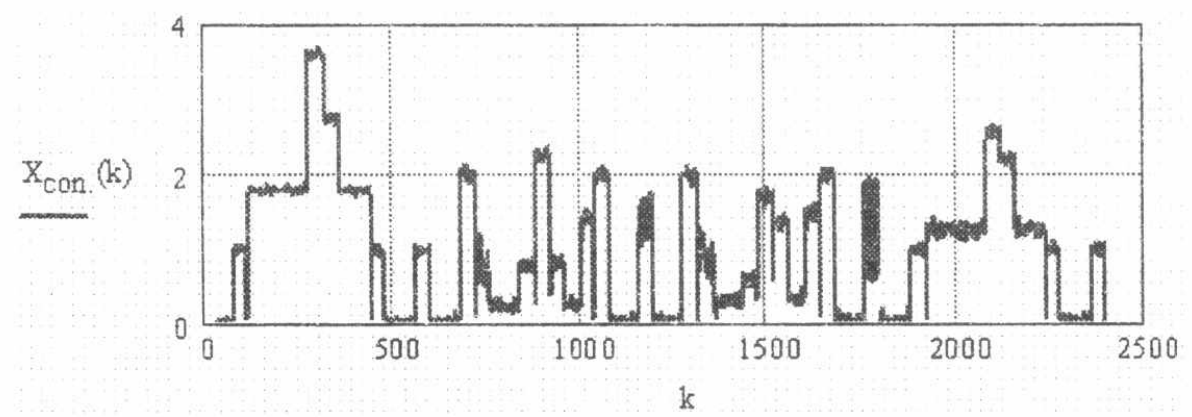

Fig.9. Single delay-line canceler output signal of the simulated conventional system. [ case (d) ] 


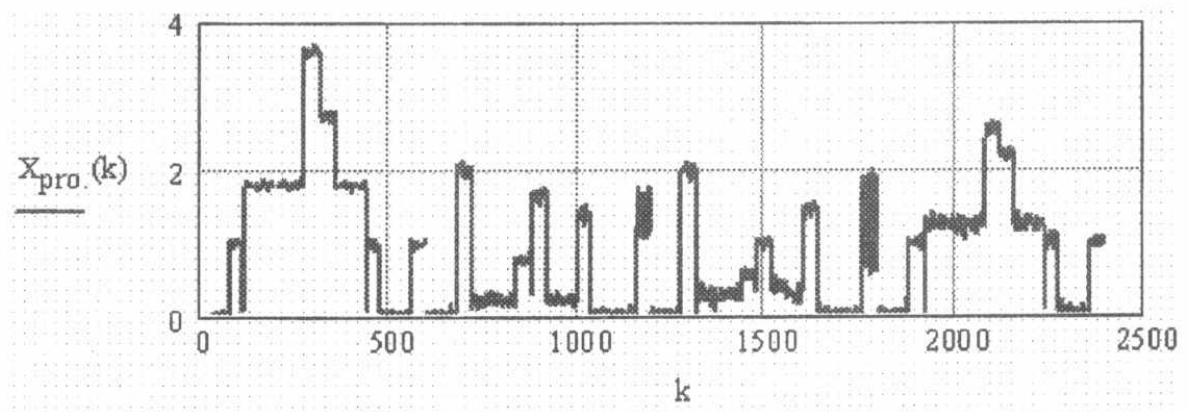

Fig.10. Single delay-line canceler output signal of the simulated proposed system. [ case (d) ] 